\title{
HEREDITARY BALL-COVERS FOR SOME BANACH MANIFOLDS ${ }^{1}$
}

\author{
JAMES E. IVEST
}

\begin{abstract}
At a problem seminar in Ithaca, New York, during January 1969, James Eells raised the question (numbered 33 on the circulated list) of whether a paracompact Fréchet manifold admits a locally finite cover by open sets, all of whose intersections are contractible. This had been established in the separable case by David Henderson, who obtained star-finite covers. This note settles the case that the model space is a Banach space homeomorphic to its countably infinite Cartesian power. The cover elements and all nonempty intersections are homeomorphic to the model. A short proof that the nerve of the cover has the homotopy type of the manifold is also included.
\end{abstract}

The purpose of this note is to observe the

THEOREM. If $E$ is a Banach space homeomorphic to its countably. infinite Cartesian power, then each paracompact E-manifold $M$ possesses a locally finite cover, closed under nonempty intersections, by open subsets each homeomorphic to $E$.

The proof of this reduces quickly to that of the Proposition below in the following way: It was shown in [8] that $M$ is homeomorphic to $K \times E$, where $K$ is some simplicial complex. ( $K$ is conceived as being embedded piecewise-linearly in a Hilbert space of suitably high dimension with its vertices members of an orthonormal basis and given the metric topology induced from the norm of the Hilbert space. In fact, the complex of [8] is locally finite-dimensional, i.e., for each vertex there is an upper bound on the dimensions of the simplices containing it.) Because all contractible, paracompact $E$-manifolds are homeomorphic [7], it is only necessary to find a locally finite, open cover of $K$ by contractible sets, all nonvoid intersections of which are also contractible.

PROPOSITION. If $K$ is a simplicial complex embedded piecewise-linearly in a Hilbert space with its tertices in an orthonormal basis and with the norm induced topology, then there is a locally-finite, open cover of $K$ by contractible open sets, all nontoid intersections of which are also contractible.

Received by the editors December 9, 1969.

AMS 1969 subject classifications. Primary 5755.

Key words and phrases. Banach manifold, locally-finite cover, nerve, homotopy type.

1 This research entirely supported by NSF grant GP-9397.

- American Mathematical Society 1972 
Proof. The open $\operatorname{star} \mathrm{st}^{\circ}(x, K)$ of a point of $K$ is the union of all open simplices of $K$ whose closures contain $x$. The symbol $K^{n}$ means the subcomplex of $K$ composed of all simplices of dimension no greater than $n$, and the symbol $K^{(n)}$ means the $n$th barycentric subdivision of $K$. (We note that the usual map $K^{(n)} \rightarrow K$ is a homeomorphism if $K^{(n)}$ is regarded as a simplicial complex embedded as a Hilbert space in the same fashion $K$ is. A proof of this appears in [4].) Now let $\mathbb{U}_{0}=\left\{\mathrm{st}^{\circ}\left(v, K^{(1)}\right) \mid v \in K^{0}\right\}$, and (inductively) define

$$
\mathscr{U}_{n}=\left\{\mathrm{st}^{0}\left(v, K^{(n+1)}\right)\left|v \in\left(K^{(n)}\right)^{0}\right| \bigcup_{j=0}^{n-1} \mathscr{U}_{j}^{*}\right\}
$$

where $\mathscr{U}_{j}^{*}$ is the union of all members of $\mathscr{U}_{j}$. The collection $\mathscr{U}=\bigcup_{n=0}^{\infty} \mathscr{U}_{n}$ is the appropriate cover of $K$.

First, $\mathscr{U}$ covers $K$. This may be verified by setting, for each complex $L$, $B(L)=\left\{\Delta \in L^{(1)} \mid(\Delta)^{0} \subset\left(L^{(1)}\right)^{0} \backslash L^{0}\right\}$ and $B^{n+1}(L)=B\left(B^{n}(L)\right)$. Then,

$$
K \backslash \bigcup_{j=0}^{n} \mathscr{U}_{j}^{*}=B^{n+1}(K)
$$

This is true because a point $x$ of $K$ is not in $\mathscr{U}_{0}^{*}$ if and only if its $K^{(1)}$ barycentric coordinates with respect to vertices of $K$ are all zero, which is a restatement of the definition of $B(K)$. If, now, for $0 \leqq m<n$ it is true that $K \backslash \bigcup_{j=0}^{m} \mathscr{U}_{j}^{*}=B^{m+1}(K)$, then $K \backslash \bigcup_{j=0}^{n} \mathscr{U}_{j}^{*}=B^{n}(K) \mid \mathscr{U}_{n}^{*}$. Since by assumption $\mathscr{U}_{n}=\left\{\mathrm{st}^{\circ}\left(v, K^{(n+1)}\right) \mid v \in\left(K^{(n)}\right)^{0} \cap B^{n}(K)\right\}$, a point $x$ is in $B^{n}(K) \backslash \mathscr{U}_{n}^{*}$ if and only if its $\left(B^{n}(K)\right)^{(1)}$-barycentric coordinate is zero with respect to each vertex in $\left(B^{n}(K)\right)^{0}$. This, though, is just the statement that $x$ is in $B\left(B^{n}(K)\right)=B^{n+1}(K)$. Thus, $K \backslash \mathscr{U}^{*}=\bigcap_{n=0}^{\infty} B^{n+1}(K)=\bigcap_{n=1}^{\infty} B^{n}(K)$. However, for any $n$-simplex $\Delta^{n}, B\left(\Delta^{n}\right)$ is an $(n-1)$-complex, so $\Delta^{n} \cap B^{n+1}(K)=$ $\varnothing$ for each $n$-simplex $\Delta^{n}$ of $K$. Hence, $B^{n+1}(K) \subset K \backslash K^{n}$ and $\bigcap_{n=1}^{\infty} B^{n}=\varnothing$, i.e., $\mathscr{U}$ covers $K$.

Second, with $K$ given the Euclidean metric, that is, in $K$-barycentric coordinates $x=\left\{t_{v}(x)\right\}_{v \in K^{-0}}, y=\left\{t_{v}(y)\right\}_{v \in K^{0}}$, and

$$
d(x, y)=\left[\sum_{v \in K^{0}}\left(t_{v}(x)-t_{v}(y)\right)^{2}\right]^{1 / 2},
$$

$\mathscr{U}$ is locally finite. (This metric is the one adopted in [8].) To see this, first note that since $K^{n} \cap B^{n+1}(K)=\varnothing$ and

$$
\mathscr{U}_{m}=\left\{\mathrm{st}^{\circ}\left(v, K^{(m+1)}\right) \mid v \in\left(K^{(m)}\right)^{0} \cap B^{m}(K)\right\},
$$

$\mathscr{U}_{n+1}^{*}$ is contained in $\mathrm{st}^{\circ}\left(\left(K^{(n+1)}\right)^{0} \backslash K^{n}, K^{(n+2)}\right)$. Also, for any complex $L$, subcomplex $L_{\mathrm{i}}$ of $L$, and vertex $v$ in $L^{0} \backslash L_{1}, \mathrm{st}^{\circ}\left(v, L^{(1)}\right) \cap \mathrm{st}^{\circ}\left(L_{1}, L^{(1)}\right)=\varnothing$. Thus, st $^{\circ}\left(\left(K^{(n+1)}\right)^{0} \backslash K^{n}, K^{(n+2)}\right) \cap \mathrm{st}^{\circ}\left(K^{r_{1}}, K^{(n+2)}\right)=\varnothing$, and any open set lying in $\mathrm{st}^{\circ}\left(K^{n}, K^{(n+2)}\right)$, misses $\bigcup_{m=1}^{\infty} \mathscr{U}_{n+m}^{*}$. 
Therefore all that is necessary is to check that for each $n, \bigcup_{j=1}^{n} \mathbb{H}_{j}$ is locally finite. Now, by its very definition, each $\mathscr{U}_{j}$ is disjoint. Further, for $x$ in $K$ and $\Delta^{m}$ the carrier of $x$, i.e., the closure of the open simplex of $K$ containing $x, \mathrm{st}^{\circ}\left(x, K^{(n-2)}\right)$ misses all elements of $\mathscr{U}_{j}$ but those defined by starring vertices which lie in $\Delta^{m}$. But, since there are only finitely many vertices in $\left(\Delta^{m}\right)^{(n)}$, at most that many members of $\mathcal{U}_{j}$ meet $\mathrm{st}^{\circ}\left(x, K^{(n+2)}\right)$, and $\mathscr{U}$ is locally finite.

Finally, all intersections of members of $\mathscr{U}$ are contractible, since if $U_{1}, \cdots, U_{n}$ are in $\mathscr{U}$ and have a nonnull intersection, there must be integers $m_{1}, \cdots, m_{n}$ such that $U_{i} \in \mathbb{l}_{m_{i}}$ and $m_{i} \neq m_{j}$ if $i \neq j$. Assume that the $U_{i}$ 's are so indexed that $m_{i}<m_{j}$ whenever $i<j$. Then because $U_{i}=$ $\operatorname{st}^{\circ}\left(v_{i}, K^{\left(n_{i}+1\right)}\right)$ for some vertex $v_{i}$ of $K^{\left(n_{i}\right)}$, it must be true that $v_{j} \in \bar{U}_{i}$ for each $j>i$, as otherwise

$$
\bar{U}_{i}=\operatorname{st}\left(v_{i}, K^{\left(n_{i}+1\right)}\right), \quad U_{j}=\operatorname{st}^{\circ}\left(v_{j}, K^{\left(n_{j}+1\right)}\right)=\mathrm{st}^{\circ}\left(v_{j}, K^{\left(n_{i}+1+\left(n_{j}-n_{i}\right) \prime\right.}\right)
$$

and (unless $\left.v_{j} \in \bar{U}_{i}\right), \operatorname{st}^{\circ}\left(v_{j}, K^{\left(n_{i}+1-\left(n_{j}-n_{i}\right)\right)}\right) \cap \bar{U}_{i}=\varnothing$. This shows that $U_{i} \cap U_{j}$ is merely the topological interior in $K$ of the star of $v_{j}$ in $\bar{U}_{i}$ with respect to the triangulation induced from $K^{\left(n_{j}+1\right)}$ and must be therefore contractible. An induction shows $\bigcap_{i=1}^{n} U_{i}$ is contractible, and the Theorem is proved.

REMARKS. (a) It is not known at present whether or not all Banach spaces of infinite dimension are homeomorphic to their countably infinite Cartesian powers, but it is known [1] that all infinite-dimensional reflexive Banach spaces are homeomorphic to Hilbert spaces and [2] that all infinite-dimensional Hilbert spaces are homeomorphic to their countably infinite Cartesian powers.

(b) David Henderson ([5] and [6]) proved this theorem in the separable case, obtaining star-finite covers.

(c) A somewhat more detailed explanation of the contractibility of $\bigcap_{i=1}^{n} U_{i}$ (in the above proof) is as follows: Since $\left\{v_{n}\right\} \cup \bigcap_{i=1}^{n} U_{i}$ is starlike about $v_{n}$, there is a contraction

$$
F:\left(\left\{v_{n}\right\} \cup \bigcap_{i=1}^{n} U_{i}\right) \times[0,1] \rightarrow\left(\left\{v_{n}\right\} \cup \bigcap_{i=1}^{n} U_{i}\right)
$$

of it to $v_{n}$ with the property that $F\left(\bigcap_{i=1}^{n} U_{i} \times[0,1)\right) \subset \bigcap_{i=1}^{n} U_{i}$. Also, because each $v_{i}$ is in

$$
\bigcap_{j=1}^{i-1}\left[\operatorname{st}\left(v_{j}, K^{\left(n_{j}+1\right)}\right) \backslash U_{j}\right]
$$

there is a homotopy $G$ from the identity map of $\left\{v_{n}\right\} \cup \bigcap_{i=1}^{n} U_{i}$ to a map of it into $\bigcap_{i=1}^{n} U_{i}$ such that $G\left(\bigcap_{i=1}^{n} U_{i} \times[0,1]\right) \subset \bigcup_{i=1}^{n} U_{i}$. (This may be constructed by first "sliding" $v_{n}$ toward $v_{n-1}$ into $U_{n} \cap U_{n-1}$, then "sliding" the image of $v_{n}$ toward $v_{n-2}$ into $U_{n} \cap U_{n-1} \cap U_{n-2}$, and so on.) Then $H(x, t)=G(F(x, t), t)$ contracts $\bigcap_{i=1}^{n} U_{i}$. 
COROllary. If $E$ is a Banach space homeomorphic to its countably infinite Cartesian power, then each paracompact E-manifold $M$ has a locally finite open cover (by topological E-balls, any nonempty intersection of which is an E-ball) whose nerve is of the same homotopy type as $M$.

Proof. It is sufficient to consider the cover $\mathscr{U}$ of the complex $K$ constructed in the proof of the Proposition and to show that its nerve $N$ is a subdivision of the complex $K_{1}$ consisting of $K$ given the weak topology, since a theorem of Dowker [3] demonstrates that $K_{1}$ is of the same homotopy type as $K$, which is (homeomorphic to) a strong deformation retract of $M$.

Therefore consider the following subdivision $S\left(K_{1}\right)$ of $K_{1}$ : For each simplex $\Delta^{n}$ of $K_{1}$, consider all $(n+1)$-tupies $\left(v_{0}, \cdots, v_{n}\right)$ of vertices of $\left(\Delta^{n}\right)^{(n)}$ such that there exists a sequence $\sigma^{n}, \sigma^{n-1}, \cdots, \sigma^{0}$ of simplices with $\sigma^{i}$ in $B^{n-i}\left(\Delta^{n}\right)^{(1)}$-here, $B^{0}\left(\Delta^{n}\right)=\Delta^{n}$-such that $\left|\sigma^{i}\right| \supset\left|\sigma^{i-1}\right|$ for $1 \leqq i \leqq n$ and $v_{i}=\left(\sigma^{n-i}\right)^{0} \cap B^{n-i}\left(\Delta^{n}\right)^{0}$. Then $\left\langle v_{0}, \cdots, v_{n}\right\rangle$ is to be an $n$-simplex of $S\left(\Delta^{n}\right)$. Now, $S\left(K_{1}\right)$ is to be the subdivision of $K_{1}$ which reduces to $S\left(\Delta^{n}\right)$ on each $\Delta^{n}$ in $K_{1}$. An alternate description of $S\left(K_{1}\right)$ is this: Let $S_{0}\left(K_{1}\right)=K_{1}$, and let $S_{n}\left(K_{1}\right)$ be defined inductively for positive $n$ to be the stellar subdivision of $S_{n-1}\left(K_{1}\right)$ which induces the first barycentric subdivision on $B^{n-1}\left(K_{1}\right)$ and introduces no new vertices off $B^{n-1}\left(K_{1}\right)$. The simplices of $S\left(K_{1}\right)$ are then those of $\bigcup_{n=0}^{\infty} S_{n}\left(K_{1}^{n}\right)$.

Now, the nerve $N$ of $\mathscr{U}$ is the simplicial complex (with the weak topology) whose vertices are the elements of $\mathscr{U}$ and whose $n$-simplices are the $(n+1)$-tuples of elements of $\mathscr{U}$ which have a nonempty intersection. Each $U$ in $\mathscr{U}$ is the open star of $v_{U}$ in $K^{(n+1)}$ for some positive $n$ where $v_{U}$ is in $B^{n}(K)^{0}$, and hence is a vertex of $S\left(K_{1}\right)$. Furthermore, each vertex of $S\left(K_{1}\right)$ is the "center" $v_{U}$ of a member $U$ of $\mathscr{U}$. Hence, the correspondence $U \rightarrow v_{U}$ is a natural isomorphism of the vertices of $N$ onto those of $S\left(K_{1}\right)$, and it remains to verify that it extends to a simplicial isomorphism.

If $\left\langle U_{0}, \cdots, U_{n}\right\rangle$ is a simplex of $N$, it has already been observed in the proof of the theorem that no two of the $U_{i}$ 's lie in the same $\mathscr{U}_{j}$ and that, assuming the set $\left\{U_{0}, \cdots, U_{n}\right\}$ to be ordered so that the corresponding $\mathscr{U}_{j}$ 's appear in their natural order, then

$$
v_{i} \in \bigcap_{j=0}^{i-1} \operatorname{st}\left(v_{j}, K^{(m(j)+1)}\right) \mid s \mathrm{~s}^{\circ}\left(v, K^{(m(j)+1)}\right),
$$

where $U_{j} \in \mathscr{U}_{n_{1}(j)}$. To demonstrate that $\left\langle v_{U_{0}}, \cdots, v_{U_{n}}\right\rangle=\left\langle v_{0}, \cdots, v_{n}\right\rangle$ is a simplex of $S\left(K_{1}\right)$, one must find simplices $\sigma^{t}, \cdots, \sigma^{0}$ of $K^{(1)}, \cdots, B^{t}(K)^{(1)}$ such that

(1) $\left|\sigma^{s}\right| \supset\left|\sigma^{s-1}\right|$ for $1 \leqq s \leqq t$,

(2) $\left(\sigma^{t-s}\right)^{0} \cap B^{s}(K)^{0} \neq \varnothing$, and

(3) $v_{i}=\left(\sigma^{t-m(i)}\right)^{0} \cap B^{t-m u(i)}(K)^{0}$. 
Now, it must be true that some simplex of $B^{s}(K)$ contains al! the $v_{i}$ 's for which $m(i) \geqq s$, the reason being that since $\bigcap\left\{\mathrm{st}^{\circ}\left(v, K^{(s+1)}\right) \mid m(i) \geqq s\right\} \neq$ $\varnothing$, there is a point $x$ in it and, for each $i$, a simplex $\Delta_{i}$ of $K^{(s+1)}$ which contains $v_{i}$ and whose interior contains $x$. However, there is only one simplex of $K^{(s+1)}$ whose interior contains $x$, so these must all be the same. But since all the $v_{i}$ 's in question are in $B^{s}(K)$, since a simplex of $K^{(s)}$ either lies in $B^{s}(K)$ or has exactly one vertex in $K^{(s-1) 0}$, and since each simplex of $K^{(s+1)}$ lies in one of $K^{(s)}$, there is a simplex of $B^{s}(K)$ containing all the $v_{i}$ 's with $m(i) \geqq s$, as was to be shown. Now, let $\Delta^{t}$ be a simplex of $K$ containing all the $v_{i}$ 's. Since any simplex of $K^{(1)}$ containing all $v_{i}$ 's must meet $\Delta^{t}$, the intersection is in $\left(\Delta^{t}\right)^{(1)}$, so there is a simplex $\sigma^{t}$ of $\left(\Delta^{t}\right)^{(1)}$ containing all of them. Since any simplex of $\left(\Delta^{t}\right)^{(1)}$ is a face of one with a vertex in $\left(\Delta^{t}\right)^{0}, \sigma^{t}$ may be chosen to have a vertex in $\left(\Delta^{t}\right)^{0}$. An induction establishes the necessary sequence $\sigma^{t}, \sigma^{t-1}, \cdots, \sigma^{0}$ satisfying (1)-(3) above so $\left\langle v_{0}, \cdots, v_{n}\right\rangle$ is a simplex of $S\left(\Delta^{t}\right)$ and herice of $S\left(K_{1}\right)$, so the vertex map extends to a simplicial one of $N$ into $S\left(K_{1}\right)$.

On the other side, if $\left\langle v_{0}, \cdots, v_{n}\right\rangle$ is a simplex of $S\left(K_{1}\right)$, then the definition of $S\left(K_{1}\right)$ gives immediately that if $U\left(v_{i}\right)=\mathrm{st}^{\circ}\left(v_{i}, K^{(m(i)+1)}\right)$, where $m(i)$ is the first integer $m$ for which $v_{i}$ is a vertex of $K^{(m)}$, then $\bigcap_{i=0}^{n} U\left(v_{i}\right) \neq$ $\varnothing$, so $\left\langle U\left(v_{0}\right), \cdots, U\left(v_{n}\right)\right\rangle$ is a simplex of $N$. The simplicial map is thus an isomorphism, and the proof, complete.

\section{REFERENCES}

1. C. Bessaga, Topological equivalence of unseparable reflexive Banach spaces. Ordinal resolutions of identity and monotone bases, Bull. Acad. Polon. Sci. Sér. Sci. Math. Astronom. Phys. 15 (1967), 397-399. MR 36 \#4321.

2. C. Bessaga and A. Pelczyński, Some remarks on homeomorphisms of F-spaces, Bull. Acad. Polon. Sci. Sér. Sci. Math. Astronom. Phys. 10 (1962), 265-270. MR 25 \#3344.

3. C. H. Dowker, Topology of metric complexes, Amer. J. Math. 74 (1952), 555577. MR 13, 965.

4. - _ Affine and euclidean complexes, Dokl. Akad. Nauk SSSR 128 (1959), 655-656. (Russian) MR 22 \#8483.

5. D. W. Henderson, Infinite-dimensional manifolds, Proc. Internat. Sympos. on Topology and its Applications, Herceg Novi, Yugoslavia, 1968.

6. - Infinite-dimensional manifolds are open subsets of Hilbert space, Topology 9 (1969), 25-33. MR 40 \#3581.

7. D. W. Henderson and R. M. Schori, Topological classification of infinite dimensional manifolds by homotopy type, Bull. Amer. Math. Soc. 76 (1970), 121-124. MR 40 \#4976.

8. J. E. West, Products of complexes and Fréchet spaces which are manifolds, Trans. Amer. Math. Soc. (to appear).

Department of Mathematics, Cornell Uiniversity, Ithaca, New York 14850 